\title{
How to Improve the Relationship between Doctors and Patients
}

\author{
Jinguo Wang \\ Department of Urology \\ The First Hospital of Jilin University \\ Changchun, China \\ wangjinguolily@163.com
}

\author{
Na Wang* (corresponding author) \\ Department of Anesthesiology \\ The First Hospital of Jilin University \\ Changchun, China \\ wangna080613@163.com
}

\begin{abstract}
This article is trying to find the major cause of this phenomenon and analyze the current doctor-patient contradiction and conflicts at a different point of view. According the literature and the current status, we prove that this problem can be relieved by system construction, including specifying the circumstances under which the rights of informed consent of patients themselves and the rights of the health care providers can both be guaranteed.
\end{abstract}

Keywords-doctor-patient relationship; medical treatment; patients' satisfaction; medical disputes

\section{INTRODUCTION}

Since the reform and opening up, along with the transformation of social structure, the transformation of economic system, especially the new medical management rules and the promulgation of the regulations on the medical accident treatment, the current doctor-patient relationship brings out the new features, can say at this stage of the doctorpatient relationship is the most nervous, since the founding of the most vulnerable period: the lack of doctor-patient trust, medical disputes and patient violence occurred continually, defensive health is widespread, and so on abnormal phenomenon.

\section{THE CURRENT DOCTOR-PATIENT RELATIONSHIP}

\section{A. Patients' need}

Patients need care and attention from the medical staff, need to be understood. Because of lack of medical professional knowledge patients, patients feel satisfied for service attitude of the evaluation of the medical staff often took it or not, the enthusiasm of the medical moral or not as a basis. Real life can be seen in some patients, although there is no cure, but still grateful to medical personnel. Also, there are patients are cured and discharged, some due to dissatisfaction with service attitude. Therefore, between doctor and patient to improve doctor-patient relationship between the humanistic concerns, also have a considerable impact [1]. With high expectations on the effects of medical diagnosis and treatment, some party of medical work and medical knowledge don't understand, think not cure is the hospital has a fault, and angry at the hospital doctors and the parties. In the current medical science and technology level, the medical staff do your utmost to treat some diseases also can only achieve a certain degree, but most of the patients and their families always to hospitals with high expectations, a patient with doctor debate when he said: "I didn't have what disease you diagnose, misdiagnosis of course." The medical staff think cure on one side and has nothing to do with the patients, health providers are granted to cure disease. It had not actively cooperate with treatment, even some patients do not take the initiative to faithfully to the medical staff stated condition, medical history, symptoms, or cooperate with treatment, in accordance with the doctor's advice in the event of accidents, medical staff do not know how to start, save time, delay treatment for patients with adverse consequences caused disputes [2].

\section{B. The doctor-patient contradiction}

There are some patients and their families in doctor-patient dispute unreasonable events. Worthy, some hospitals also appear near the planning of square riot "cure", poses a serious threat to hospital order. Some patients and their families do not observe the rules and regulations of the hospital. Some patients without authorization from the court or take other treatments, also hold surprises. Establish and improve the basic medical insurance system. Health insurance is the theory basis of disease risk theory. Due to the uncertainty of the individual, anyone can't know when he is in illness or injury, so you need to try to avoid the risk of disease. But group disease probability is predictable, which is the foundation of the medical insurance rate measurement [3]. The treatment of diseases tend to produce the high cost, and make the individual, family or group, so the economic losses. Medical insurance system through to every individual in a particular crowd charge lower premiums, can guarantee a certain individual in the crowd when the disease of medical fees have been paid, even if this charge amount is much higher than the payment of premiums. So, by medical insurance system, the participants as individuals, disperse the risk, reduce the suffering from disease and the economic burden. The need for medical insurance system which is the need of the whole social members, reflects the common interests of all social members, so it is a kind of public demand. The need of the public does not meet or is unwilling to provide [4]. So in order to meet the needs of the public, the government must provide the public goods of basic medical insurance system. Second, the public needs is not to the people status and income is limited, whether poor or rich people enjoy this kind of to meet the needs of the public and provide public goods are completely equal, and equal 
opportunity, and this is why the basic medical insurance as public goods provided to each person, one of the reasons for promoting the equal basic medical services.

\section{FACTORS CONTRIBUTING TO THE CONTRADICTION}

Urban workers, urban and rural residents, due to the different identity with the medical security level is different, also can not be fully equal, but it is a basic implementation, for the first time everyone will have access to health care, especially close the bankrupt enterprise retiree also enjoy the medical insurance for enterprise employees and difficulties. Close the bankrupt enterprise retiree and difficulties for enterprise employees belong to "the most adverse social members", the burden of medical expenses is most sensitive, is also an important factor of the doctor-patient relationship nervous. This group should be brought into the system of medical treatment insurance, helped improve the doctor-patient relationship, promote social harmony [5].

This suggests that the medical cost of losses mainly rely on the drug income and national finance income subsidies to compensate. At the same time, the medical staff of medical service income is far lower than the instruments and equipment checking income, objectively affect the enthusiasm of the medical staff. The hospital will have to increase the drug income in order to survive and development. In order to increase the drug income, hospital, under the same curative effect under the condition of natural medicine tends to sell you. Therefore, it is necessary to cancel the pricing mechanism, looking for a new attack intervention service compensation mechanism to replace. Cancel the mechanism of drug addition, helps to reduce between the senses of social role conflict. Medical institutions can not economic burden and the medical staff. As a result, medical personnel can be absorbed in the medical behavior, don't have to play "the unknown drugs salesman" xi social role, reduce social role conflict between doctor and patient.

In the current medical dispute processing mechanism, set up independent of the medicine, loyal to both sides, and different from the third party institution of judicial organs, the medical dispute mediation, arbitration, is a dilemma may crack doctor-patient dispute processing way [6]. Patients can draw lessons from consumer rights and interests' protection association or other social group organization model, build a similar such as patients' rights and interests protection law "and other laws and regulations, strengthen the protection of the rights and interests of patients of. Modeled on regulations on consumer groups, patients in medical disputes association can help patients social organization, the performance of the duties include providing consumer information and consulting services. To assist patients start, doctor-patient consultation, administrative mediation application medical accident identification and judicial authentication: participating in the relevant administrative departments for supervision and inspection of the medical services, etc.

\section{THE IMPACT OF MEDICAL DISPUTE ON THE SOCIETY}

Qualitative belong to social organizations, the association members need to have more professional medical background of law knowledge, skilled negotiation skills and communication skills. Association of patients can become the unequal situation, medical information of patients' rights groups, for reasonable solution to medical disputes have more professional platform and dredge. Lessons from the insurance risk sharing mechanism, set up the medical insurance, bear is not caused by medical staff negligence of medical injury consequence, such as medical accident, patients with special physical fitness and special disease, force majeure, etc. Only by establishing a certain mechanism system of professional risk can make medical workers to continuously enterprising, conquer medical blind spots and errors. This can reduce the burden of medical institutions and medical personnel, promoting the progress and development of the medicine.

Doctors should truthfully explain the patients' conditions to the patients or their family members, but should pay attention to avoid adverse impact on patients. Experimental clinical treatment, doctors shall be approved by the hospital and the consent of the patient or family members. Surgery medical institutions, special inspection or special treatment, must be patient consent, and shall obtain the families or related parties agree and sign. The patients cannot be obtained, as families or related parties agree and sign.

\section{THE SUGGESTED SOLUTIONS}

Unable to obtain the patient advice and have no family or participant is present, or some other special circumstances, the doctors should cure medical treatment scheme is put forward, in the medical institution or the authorized responsible for personnel implementation after approval. A medical institution shall respect patients about their condition, diagnosis, treatment of knowledge rights. In operation, special inspection, special treatment, make necessary explanation shall be submitted to the patients. Because of medical protective measures should not be informed to the patients and shall notify relevant situations to the patients' families."

\section{A. Fully carrying out informed consent}

In the professional medical act, the main body of informed consent is patients and patients' families. In the "measures for the administration of medical institutions", informed consent of people outside the patients and patients families, adds to the party. This is caused in the process of medical practices and patients in a matter of fact may not be able to exercise their right to know, in an awkward position. At the same time, it also leads to cure is difficult to determine for object, objectively also increased the difficulty of the implementation of informed consent. Therefore, we should strengthen the legislation specify the circumstances under which the right to informed consent by patients themselves, the circumstances under which shall be exercised on his behalf by family members or party.

Second, the law should be clear in all cases, adopt what kind of way of informed consent. In the relevant laws and regulations, we see only during surgery and experimental medical, only regulations must obtain the signature of the patient or family members, other cases what way, informed consent is informed orally or notify, and there is no clear statement [7]. For patients, it is obviously unfair. Therefore, we 
should strengthen the legislation, to ensure that patients receive the corresponding civil compensation. In the course of the treatment, should have corresponding medical instrument to protect the informed consent right of patients. Inform also shall timely. When, in the process of surgery or other medical emergency needs to change the original treatment, should inform the patients or their families, get approval rear can continue to treatment.

Doctors should truthfully explain the patients' conditions to the patients or their family members, but should avoid adverse impact on patients. Provisions of regulations on the handling of medical accidents, medical institutions and medical personnel were informed application, when to avoid adverse impact on patients. This is the so-called "protective medical treatment". Protective medical and informed consent, there is a certain contradiction. This requires medical institutions or medical personnel, in the performance of the inform procedure, should with patient benefit as the basic starting point, adhere to the "do not harm patients," the basic ethical principles.

\section{B. To prevent medical malpractice}

Technology and quality is the hospital survival and the development condition in the aspect of improving medical personnel professional quality, first of all attach great importance to the training of medical personnel. Followed by paying special attention to the medical quality management, improve the system of all kinds of medical management. Develop appropriate work standard and system, and ensure the system to use [8]. The aim is to upgrade the overall quality medical personnel, establish a good quality, good professional ethics and self-supervision spirit, good professional quality, finally set up service concept for the center with the patient.

It is a high-risk occupation health work, medical personnel legally unable to bear the liability for this risk, should strengthen the early to inform, let patients know should not only accept the medical service, to accept risk may be damaged. Patients agree to work is the medical tort liability is necessary conditions, is legitimacy medical work behavior under the premise of medical staff should be the purpose of each operation, risk factors, to inform the patient and family, for special treatment, nursing, inspect the consent of the patients, signing formalities when necessary, it is respect for the rights of patients is the need of medical workers to protect themselves [9].

\section{To Manage medical records in a proper way}

In daily life, there are a lot of medical disputes are caused by the lack of doctor-patient communication, therefore, we must strengthen the doctor-patient communication, to establish a good doctor-patient relationship, advocated the spirit of loving, must strengthen the implementation of the new medical model and the establishment of humanistic spirit, to persistent education of medical ethics and medical personnel, education they firmly establish a "taking patients as the center, the patient first" idea, and respect the rights of patients, and improve service, take the initiative to establish equal relationship with the patient. Strengthen the doctor-patient communication, is to reduce medical disputes, an important means to ease the doctor-patient relationship.

So the doctors need to master to comfort the patient's facial expression, control will cause patient expression of misunderstanding. Pay particular attention to is in the process of doctor-patient communication: when seeing patients, doctors may not happy mood, but his bad mood can never be expressed through the eyes and facial expressions to patients. This is a doctor must have the professional quality.

Medical file is the evidence that medical personnel clinical think, is the original record in the process of diagnosis and treatment, there is documentary evidence. In medical disputes, is a technical appraisal, the basis of judicial authentication, distinguish responsibility [7]. In the event of dispute, if the medical file is incomplete, often makes the parties in a passive position and assume no responsibility. Therefore, various medical documents writing should be in accordance with the relevant regulations, laws and regulations and preservation, shall not be arbitrarily changed, destroyed. In the case when a change is to be more careful, should be described in detail in the course of records in chronological order, shall not be altered or inserted in the medical record writing. According to the dialectical materialism viewpoint, internal cause is based on, external cause is conditions. External cause by internal workers and at the same time can also be an organic part of gradually into the internal cause.

\section{CONCLUSIONS}

Establish and improve the basic medical insurance system, establishing the mechanism of the third party to deal with disputes, to provide guarantee for medical institutions and medical personnel, perfect the legislation safeguard patients right of informed consent, to establish a good doctor-patient communication, strengthen medical quality management to reduce medical negligence are helpful to the improvement of the doctor-patient relationship.

\section{REFERENCES}

[1] K Qin, L Li, HQ Wang. Differences in cognition between doctors and patients and medical disputes. Medicine and Society. 2007

[2] DAVID A. KENNY, WILLIA MCOOK. Partner effects in relationship research: Conceptual issues, analytic difficulties, and illustrations[J]. Personal Relationships. 2005 (4)

[3] Edward A. Walker, Wayne J. Katon, David Keegan, Gregory Gardner, Mark Sullivan. Predictors of physician frustration in the care of patients with rheumatological complaints[J]. General Hospital Psychiatry. 1997 (5)

[4] Haroll Balme. China and Modem Minicine-A Study in Medicine Mission- ary Development. 1921

[5] SA M. McLean. A Patient's Right to Know: Information Disclosure, the Doctor and the Law. 1989

[6] Mccoid. The Care Required of Medical Practitioners. Vand. L.Rev. 1959

[7] R. Nicole, "Title of paper with only first word capitalized," J. Name Stand. Abbrev., in press.

[8] Y Yorozu, M Hirano, K Oka and Y Tagawa, "Electron spectroscopy studies on magneto-optical media and plastic substrate interface," IEEE Transl J. Magn. Japan, vol. 2, pp. 740-741, August 1987 [Digests 9th Annual Conf. Magnetics Japan, p. 301, 1982].

[9] M Young, The Technical Writer's Handbook. Mill Valley, CA: University Science, 1989. 